\title{
Extended roles for allied health professionals: an updated systematic review of the evidence
}

\author{
This article was published in the following Dove Press journal: \\ Journal of Multidisciplinary Healthcare \\ 13 October 2014 \\ Number of times this article has been viewed
}

\author{
Robyn L Saxon ${ }^{1-3}$ \\ Marion A Gray ${ }^{1,2}$ \\ Florin I Oprescu ${ }^{1,2}$ \\ 'School of Health and Sports Sciences, \\ Faculty of Science, Health, Education \\ and Engineering, ${ }^{2}$ Cluster for Health \\ Improvement, University of the \\ Sunshine Coast, Sippy Downs, QLD, \\ ${ }^{3}$ Queensland Health, Brisbane, QLD, \\ Australia
}

Background: Internationally, health care services are under increasing pressure to provide high quality, accessible, timely interventions to an ever increasing aging population, with finite resources. Extended scope roles for allied health professionals is one strategy that could be undertaken by health care services to meet this demand. This review builds upon an earlier paper published in 2006 on the evidence relating to the impact extended scope roles have on health care services.

Methods: A systematic review of the literature focused on extended scope roles in three allied health professional groups, ie, physiotherapy, occupational therapy, and speech pathology, was conducted. The search strategy mirrored an earlier systematic review methodology and was designed to include articles from 2005 onwards. All peer-reviewed published papers with evidence relating to effects on patients, other professionals, or the health service were included. All papers were critically appraised prior to data extraction.

Results: A total of 1,000 articles were identified by the search strategy; 254 articles were screened for relevance and 21 progressed to data extraction for inclusion in the systematic review.

Conclusion: Literature supporting extended scope roles exists; however, despite the earlier review calling for more robust evaluations regarding the impact on patient outcomes, costeffectiveness, training requirements, niche identification, or sustainability, there appears to be limited research reported on the topic in the last 7 years. The evidence available suggests that extended scope practice allied health practitioners could be a cost-effective and consumeraccepted investment that health services can make to improve patient outcomes.

Keywords: allied health professionals, extended scope practice

\section{Introduction}

Worldwide there is a trend towards an ageing population. ${ }^{1}$ People are living longer, and subsequently there are higher levels of chronic disease. ${ }^{2-4}$ Health care services must operate with finite resources. Trends focused on funding increases are widely considered unsustainable. ${ }^{5,6}$ An international survey of eight industrialized countries described difficulties in terms of cost per patient and patient outcomes for each of the different health care systems. Due to the larger proportion of health funding for this age group, the survey focused on chronically ill patients. This research reported issues with access, care coordination, and efficiency (ie, duplication, patient perceptions of wasteful care), safety in terms of outside hospital errors, and processes to support patient self-management. It concluded that many of the participating countries were shifting resources from managing the traditional acute care to the more long-term chronic care required for the changing population demographic. ${ }^{4}$ 
Many foci for improvements around these issues have been reported in the literature, such as standardization of discharge and transition processes, ${ }^{2}$ technological innovations and their correlation with level of health care, ${ }^{7,8}$ and extended scope roles for non-medical practitioners. ${ }^{9-18}$

Traditional models of health service are generally managed around the needs and capabilities of individual disciplines, with medical practitioners considered the key decision-makers in aspects of referral, admission, and discharge. With limited specialist resources, this often equates to a long wait for service for patients, even for those patients who may benefit from early allied health input. ${ }^{15,18-21}$ Thus, allied health practitioners have been identified as possessing the key clinical skills and capability to bridge this service demand gap and act as the first point of contact within the patient's health care journey. ${ }^{10,20-25}$ In essence, to improve patient access to timely health care, new extended scope allied health roles are being trialed with clinicians accessing specialized training outside the traditional scope of their discipline. This may involve identified tasks traditionally undertaken by medical, nursing, physiotherapy, occupational therapy, speech pathology, or other health professionals. A systematic review published in 2006 found variability in the use of terminology, and focused on proposing a working definition of extended scope of practice in terms of enhancement and substitution (enhancing job depth by adding skills within a profession and expanding job breadth by working across professional boundaries). ${ }^{15}$ In addition, this review highlighted that there was indeed support for introducing these roles; however, the majority of the publications at that time focused on reducing waiting times (and the burden on medical practitioners), with limited inclusions regarding the impact on patient outcomes, cost-effectiveness, training requirements, niche identification, or sustainability.

The aim of the current paper is to build on the aforementioned review by including recently published evidence relating to the effects these roles have on patient care, other professionals, and the health care service.

\section{Materials and methods}

This review updated and expanded on the evidence collected in a previously published systematic review by McPherson et al in 2006. ${ }^{15}$ Hence the search strategy was limited to the years 2005-2013 and followed the three-part PICO approach, ie, (P) patients/professions, (I) intervention, (O) outcome, omitting the (C) controls. The electronic databases PubMed, Embase, and CINAHL were searched using the full search terms described in the complete report by
McPherson et al (see Supplementary material). ${ }^{16}$ Assistance from an experienced health librarian was used to ensure the strategy was implemented correctly. Following the initial computer-based search, there was one modification of the abstract screening process used by McPherson et al. The original review included five allied health groups based on the experience of the research team (physiotherapy, occupational therapy, speech pathology, paramedics, and radiography). ${ }^{15}$ Due to the perceived synergies of the roles within the health care system and patient contact point, only articles relating to physiotherapy, occupational therapy, and speech pathology were considered for inclusion in this current review. Paramedics and radiographers were excluded as these professions tended to work in more discrete patient contact areas, with extended scope relating almost exclusively to traditional medical roles. Therefore, only papers relating to extended scope practice (ESP) or advanced scope of practice for allied health practitioners (Adv HP), physiotherapy, occupational therapy, or speech pathology were screened. The other variance to the search strategy of McPherson et al was the exclusion "gray" literature, due to this literature not being able to progress to the critical appraisal point. ${ }^{15}$

All remaining titles and abstracts were screened for relevance by the first author; those abstracts then had their relevance confirmed or were deemed not relevant by the other two authors prior to full-text review and data extraction. Duplicate studies were excluded. Abstracts from conference proceedings were excluded (unless the published article was obtainable). Only English text versions were included. The full texts of 55 studies were retrieved and reviewed to confirm relevance and for possible data extraction. The reference lists and citations of these publications were hand-searched for further possible relevant articles. An additional five articles were included via this process.

Quality screening for data extraction followed the criteria outlined by McPherson et al (based on the Critical Skills Appraisal Skills Programme, the Centre for Reviews and Dissemination at York). ${ }^{16}$ Articles were screened for relevance and the presence of qualitative or quantitative data relating to ESP within the three professions by all three authors. All studies that reported data were included. Studies that were narrative with no data presented were excluded.

Studies that described traditional roles (not extended scope) were excluded. Evidence quality was designated based on the National Health and Medical Research Evidence Hierarchy. ${ }^{26}$ Due to the heterogeneity of the studies in both design and participant numbers, no meta-analysis was attempted. 
An Excel spreadsheet for data extraction was compiled by the first author and subsequently reviewed and amended by two other authors.

\section{Results}

The initial search yielded 1,000 articles. Following abstract screening for potential relevance $(n=254)$, the full texts of 55 articles were retrieved. An additional five articles were identified via searching of the reference lists or citations of the selected articles. Hence, a total of 60 full-text articles were reviewed for data extraction. Data extraction was not considered for 39 of these articles as these were literature reviews, duplicates, conference papers, editorials, commentaries, not relevant, or descriptive only (Figure 1).

Most of the articles (including the reviews) related to physiotherapy ESP only ( $\mathrm{n}=19)$, with five remaining articles including physiotherapy as part of a multiple discipline study. Only one article had data related to occupational therapy ESP. Data relating to ESP and speech pathology were found in the multiple discipline study and the systematic reviews. These articles are summarized in Table 1.

\section{Results by discipline}

\section{Physiotherapy}

The majority of literature related to physiotherapy ESP, which is similar to the findings of McPherson et al; however, there were no randomized controlled trials as originally recommended. The literature included cohort studies, ${ }^{20-22,27-31}$ surveys reporting tasks undertaken, ${ }^{12,32-38}$ and a number of systematic reviews relating to orthopedic

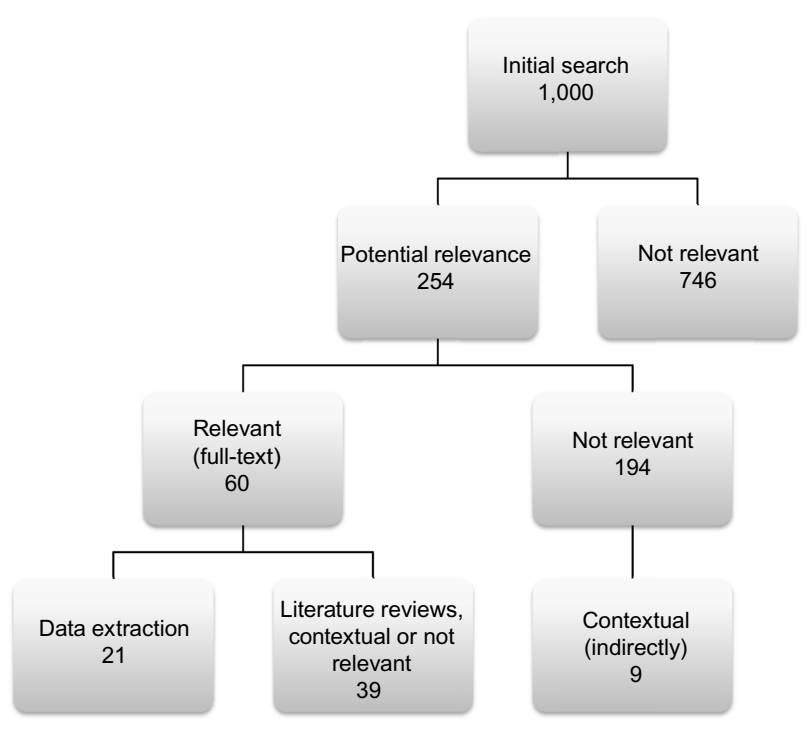

Figure I Stages of search strategy results. outpatients ${ }^{13,18,39}$ or inflammatory conditions. ${ }^{40}$ Only one article reported on a pre-post impact that included patient outcomes. ${ }^{28}$ This described physiotherapists working within an orthopedic clinic, recommending conservative management for non-surgical patients, and reported improvements in patient outcome scores (chronic disease self-efficacy scores); however, no comparison was made with the surgical patient group or non-surgical patients who received no physiotherapist intervention (only orthopedic consultation). Further, there is no clarity in this article as to whether this outcome constitutes ESP for physiotherapy or merely full scope of practice? Indeed, one review conducted by Stanhope et al as a follow-up on ESP physiotherapy practice in orthopedics reported a lack of clear differentiation between extended scope and full scope practice, a continued paucity of research into the area, and that most studies are of a lower quality on the National Health and Medical Research Council. ${ }^{18}$ Further, Anaf and Sheppard proposed in their 2007 review that in the emergency department context, the reported tasks undertaken are within scope and are there for "advanced" (or full scope) and not "extended" scope of practice. ${ }^{41}$

A number of studies described tasks undertaken by ESP physiotherapists, including: triaging, referral to other services (including medical specialists), requesting investigations (radiology, pathology), educational resources, diagnostic ultrasounds, medication (limited prescription, monitoring, dosage changes), joint injections (recommend or perform), removing K-wires, simple suturing, and prescribing conservative management/treatment/exercises. ${ }^{12,13,15,18,29,37,40}$

Some of these tasks are not ESP in all contexts, with some performed under the oversight of a medical officer, some relating to funding restrictions dependent on legislation, and others related to uptake of policy. ${ }^{12,15,21,22,41,42}$ Indeed, as some authors have pointed out, due to legislative changes, what was once considered ESP for a profession becomes within scope, hence adding to the difficulty in definition of ESP for research purposes. ${ }^{11,15}$

It appears that physiotherapy ESP research is focused on direct access for musculoskeletal conditions (USA and Scotland), inflammatory conditions (Canada and Australia), and orthopedics (UK, Canada, and Australia). There are some studies relating to physiotherapy practice in the emergency department; however the consensus appears to be that this is not ESP, merely a different context. ${ }^{23,41,42}$

\section{Occupational therapy}

Similar to the findings of McPherson et al, studies relating to occupational therapy extended practice that met the criteria 


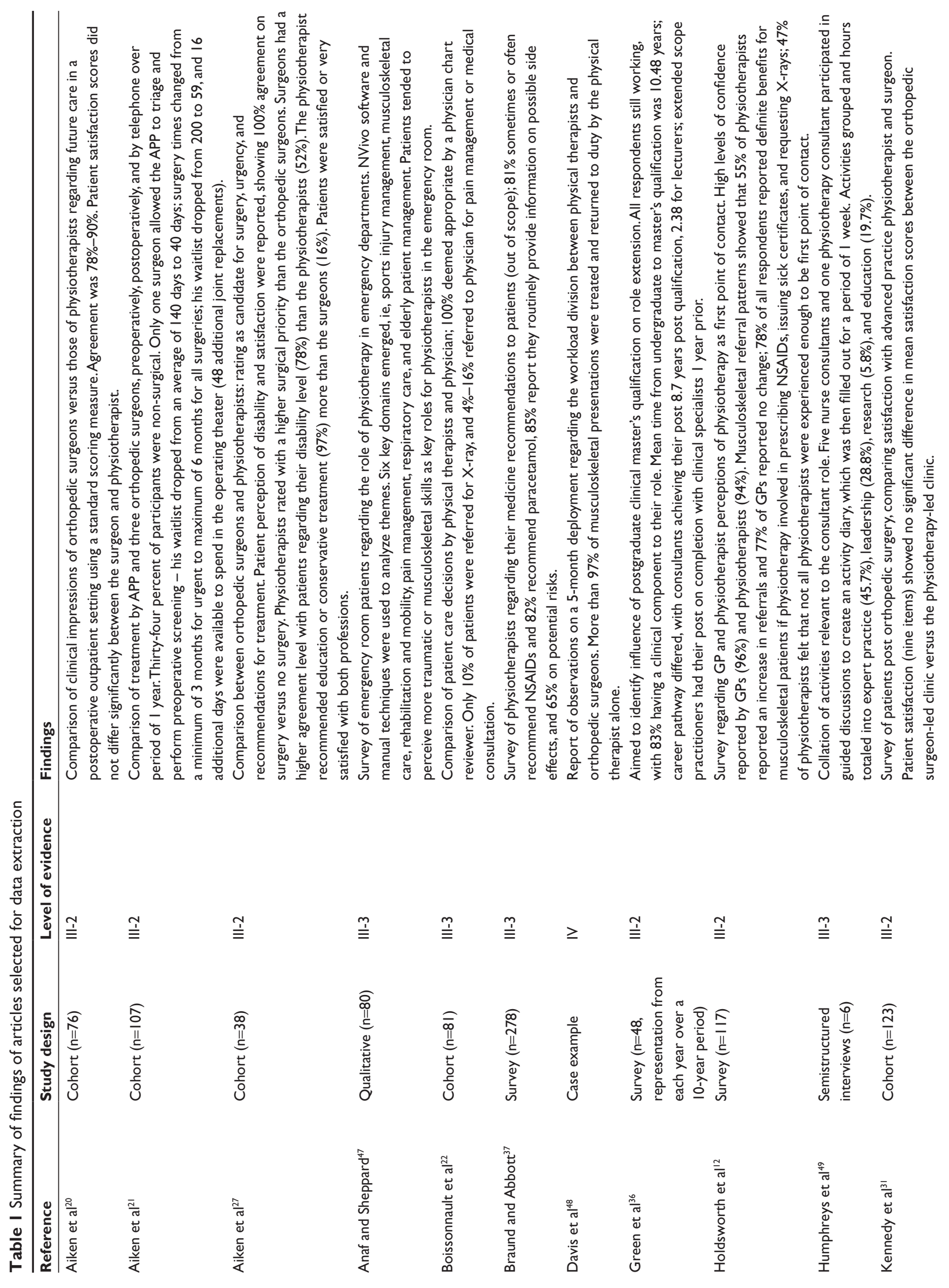



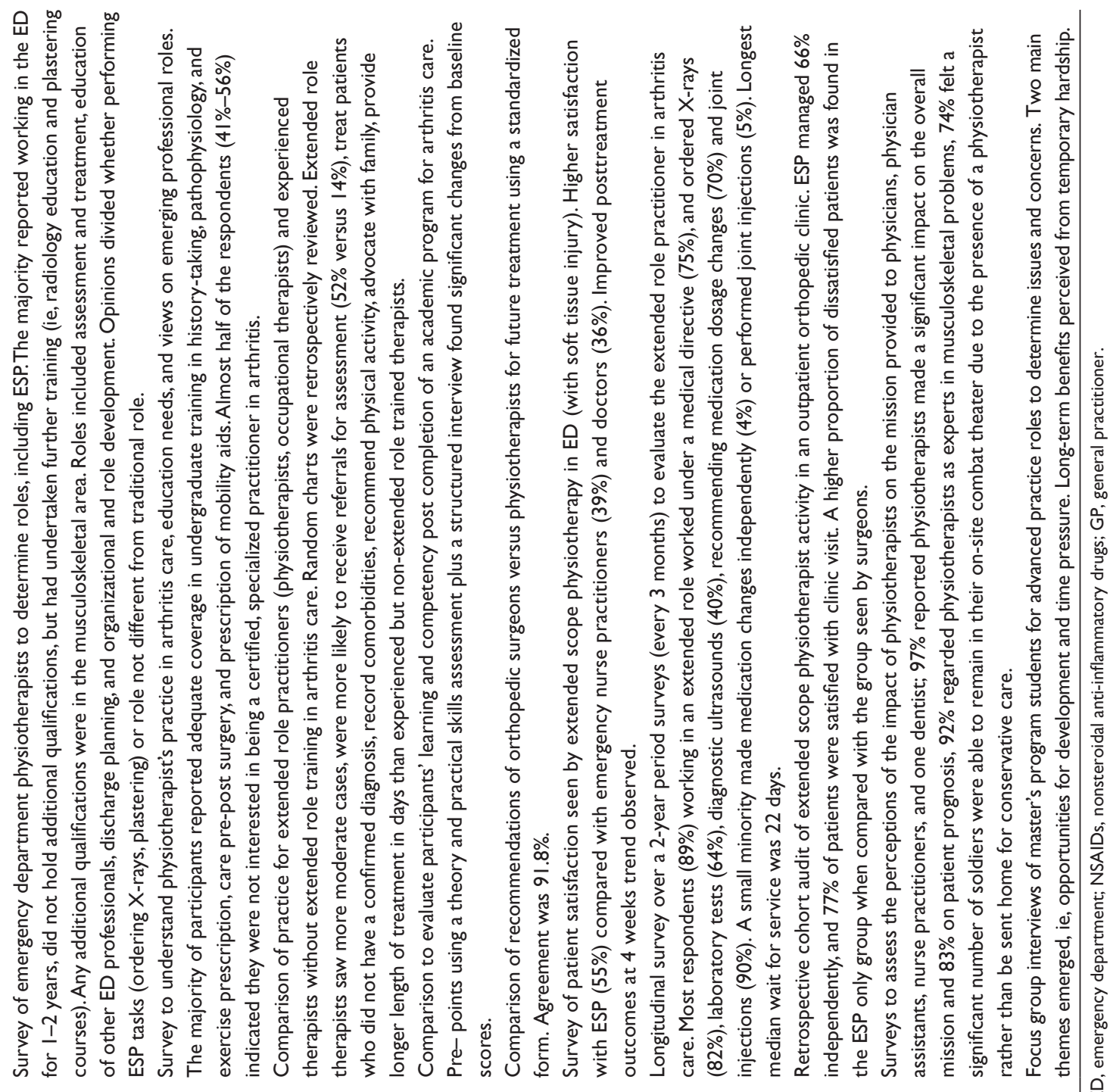

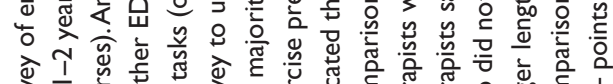

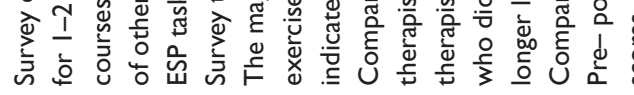

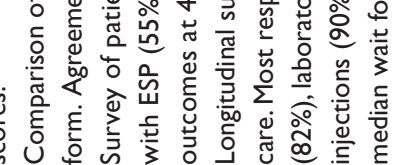

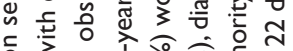

ळ

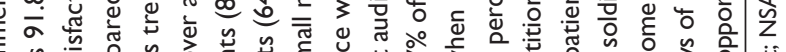

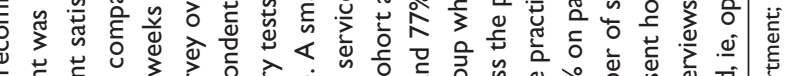

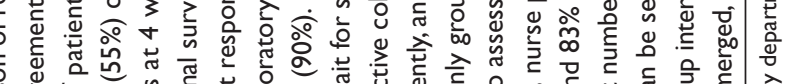
उ

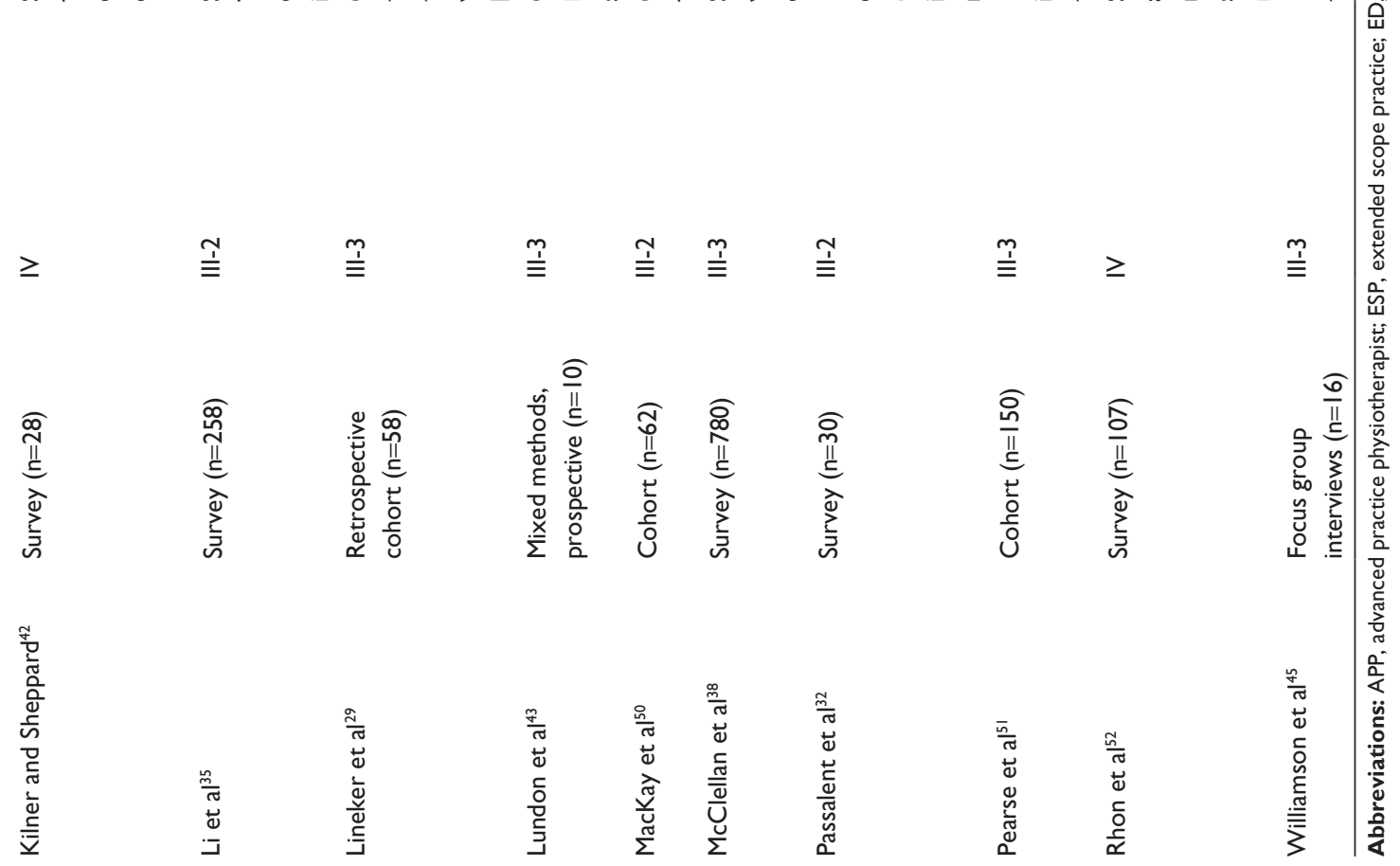


included both physiotherapists and occupational therapists. Three of these articles centered on a specific program for arthritis care. ${ }^{29,32,43}$ The studies explored the change in clinician knowledge following training, initially utilizing a practical skills assessment in addition to theory testing. This was followed up via a survey for 2 years to evaluate the impact of the training on practice and the tasks undertaken. It was reported that whilst the majority worked in extended scope roles and were able to order X-rays, laboratory tests, and ultrasounds, three quarters still worked under a medical directive. ${ }^{32}$ Patient outcomes were explored in terms of type of referral, treatment provided, and length of treatment. No data were reported on patient improvement or reduced waiting time to treatment. In addition, the study numbers were relatively small $(n=58, n=30$, and $n=10)$ in comparison with the physiotherapy data (see Table 1).

One article related directly to occupational therapy ESP that did not make data extraction was a military case study that described the training, credentialing process, and tasks undertaken by an ESP. ${ }^{44}$ The training process included formal coursework coupled with a period of internship, ie, a model similarly described for ESP within other professions. Tasks included direct access, limit duty, referral to specialist clinics, triaging, and conservative management/treatment. The article aptly describes the role as a "force multiplier", an underpinning objective stated for all ESP roles.

\section{Speech pathology}

Speech pathology ESP was included in articles surveying perceptions of professionals undertaking postgraduate studies, and within other published systematic reviews. ${ }^{15,45}$ No further specific studies were found outlining speech pathology extended scope trials that were included for data extraction. One article described the tasks and training undertaken by speech pathologists working within a neonatal intensive care unit; however, no data were included. ${ }^{46}$ This may indicate the infancy of this profession in extended scope role development.

\section{Discussion}

There continues to be high levels of interest in extended scope roles; however, the quality of evidence relating to their impact remains low and not inclusive of areas highlighted for future research by McPherson et al in 2006. The highest number of published articles by far was within the physiotherapy profession, with almost all relating to the treatment of orthopedic patients or those with inflammatory arthropathy. A number of systematic reviews have been published for specific physiotherapy areas of practice, with all discussing the low level of evidence found. ${ }^{13,15,18,39,40}$ In comparison, the occupational therapy and speech pathology professions appear to be in their infancy of extended scope roles, with only a few published articles identified and suitable for inclusion. ${ }^{15,43-45}$

It appears that the majority of the articles that describe outcomes in terms of reduced waiting times, do not consistently specify to which point in the patient journey - the Adv HP role or the medical profession or indeed both. Neither do they comment specifically on the proportion of patients who do not progress to medical specialist appointments following input from the Adv HP. One article mentioned that a percentage of patients did not need to see the surgeon. ${ }^{21}$ Both of these measurements would be integral in measuring cost-effectiveness, in terms of the health organization and the patient. Reduced cost to the patient in terms of travel to the health care facility, time away from work, and changes in quality of life related to earlier intervention. The most commonly cited method for measurement of cost-effectiveness at an organizational level relates to differences in wages paid to treat a patient (eg, Adv HP AU\$58 versus medical specialist AU\$128). ${ }^{18,22,39}$ The potential cost savings encompassing the impact on reducing the number of appointments is often not factored; frequently, the medical specialist orders tests and/or treatment from an allied health professional and then books a review with the patient. ${ }^{20}$ Additionally, the benefits to the health service may be realized further afield, in terms of increased medical specialist availability to acutely ill patients, surgical lists, or highly complex cases. ${ }^{12,20,21,27,48,52}$ By changing the way a patient enters the service, ie, having the Adv HP request routine information (pathology, radiology, treatment) ready for the first medical specialist appointment, may achieve cost benefits for both the health service and the patient in terms of a reduced number of appointments overall. The same may hold true for collection of routine follow-up information. Indeed, one study by Aiken et al in 2009 quantified this in terms of additional 16 days in the operating theater performing an additional 48 joint replacement procedures. $^{21}$

\section{Practical implications and future directions of research}

Internationally, a number of innovative workforce redesign projects have explored the ability of allied health professionals to extend their scope of practice beyond traditional boundaries, for example, physiotherapists in an 
emergency department, physiotherapists in orthopedic clinics, physiotherapists and occupational therapists in arthritis care, occupational therapists in upper extremity management, and speech pathologists in fiber optic endoscopy. ${ }^{13,15,18,32,39-41,44}$ Reports of additional skills acquired include increased clinical reasoning, ordering and interpretation of diagnostics (radiology, ultrasound and pathology), limited medication management (prescription, dosage changes, monitoring), and procedures (joint injections, passing endoscopes, removing K-wires, applying plaster of Paris, simple suturing). Changes to health care practice included first point of contact (direct access), additional context of care (emergency department, orthopedic triage), and an increased role in education of both patients and other clinicians. ${ }^{12,22,27,29,36,38,41,49}$ The majority of these extended scope roles were implemented in the context of increasing patient demand, with varying levels of evaluation and use of terminology to describe the role. It appears that the majority of the literature focuses on reducing patient waiting times to medical specialist appointments (particularly for surgery) and comparing the categorization of these patients by advanced or extended scope allied health practitioners with that of their medical colleagues. ${ }^{20,21,27,30,48,50}$ There remains limited evidence as to the true impact in terms of overall patient waiting times. In fact, the majority of the literature describes these comparisons in terms of a retrospective audit or as a simultaneous clinical pathway. This appears to be the "groundwork" to convince health care professionals and managers that roles can be substituted. Only one study reported on the difference between one model of care and another. It found that the surgeon's waitlist dropped from 140 to 40 days, his overall waitlist dropped from 200 to 59, and an additional 16 days were available to operate, with 48 additional surgeries performed. ${ }^{21}$

There was rudimentary discussion relating to the cost benefits this created for the health care service or the patients. All of the studies reporting on patient satisfaction found no difference between the allied health profession and the medical practitioner. ${ }^{20,21,27,31}$ In fact, one study reported patients valuing the additional time allied health practitioners spent in comparison with their medical colleagues. ${ }^{47}$ However, one study reported that although patient satisfaction was $77 \%$, a higher proportion of dissatisfied patients were in the group seen by an extended scope practitioner only. ${ }^{51}$

Some of these roles have been described as advanced practice or practice in a non-traditional context, rather than as extended scope. Strong images of these roles on a continuum, such as the "skills escalator" described by Gilmore et al, as practitioners gain skills within their discipline to a highly skilled or "advanced level" then undertake training to develop additional skills to extend their scope of practice. ${ }^{11}$ Another strong image comes from the military context, where acquiring skills outside traditional roles through structured training and credentialing programs are described as a "force multiplier". ${ }^{4}$

There appears to be emerging agreement regarding particular tasks that are considered extended scope, such as ordering and interpreting of plain film X-rays, limited prescribing rights, limited ordering of pathology tests, and specific injection tasks. ${ }^{13,15,18,40}$

Additionally, clarity around the context in which care is delivered does not necessarily mean the role involves extended scope practice. It is becoming more common to see these context-dependent roles described as advanced scope practice, such as physiotherapists working within musculoskeletal, orthopedic, and emergency pathways of care, and occupational therapists or physiotherapists working with arthritis. ${ }^{21,23,25,28,38,40,42}$

Nine systematic literature reviews were found using the search strategy, the majority related to physiotherapy practice. All authors commented on the low quality of the research (with hierarchy ranging from II to IV) and a lack of improvement in the research over time. ${ }^{11,13,15,18,39-41,53}$ Initial searches reported varying numbers of articles, from approximately 150 to over 1,000; however, in all literature reviews, the majority of these were not included, in general less than 12. All authors commented on the positive reports, and outlined tasks performed by the roles, a high level of patient satisfaction, and impacts on process measures such as waiting times; however, they highlighted a lack of "robust data" in terms of patient outcomes, including effectiveness and information on specific training programs. The training programs described include ad hoc "on the job" training, specialized training programs with credentialing, and the development of more formalized tertiary masters level courses. It appears clear that the consensus is for a structured training environment with regular ongoing support, whilst maintaining a strong focus on independent practice.

\section{Future research}

As evidence is building, albeit at a low level from an academic perspective, it appears that patient access to care is improving, patients are satisfied with the care provided, and health care decision-makers are continuing to invest in establishing and trialing these new roles. It is acknowledged that there may be studies that were not found through this search or pragmatic evidence contained in the gray literature that contributes to 
this dialog. The challenge remains in terms of legislative barriers and inconsistencies. These barriers feed into maintaining the status quo, with a power differential towards the medical fraternity, with most extended scope practitioners still requiring a direct physician referral or to work under a "medical directive". 21,22,32,42 This exact bottleneck of all patients requiring specialist medical consultation prior to receiving care in conjunction with overworked junior doctors ${ }^{10,13,15}$ in addition to a shortage $e^{25,27,35}$ was described as a precursor to the implementation of trials of extended scope health practitioners.

So it seems that allied health practitioners can undertake training to appropriately learn and perform new skills outside the traditional scope of their practice, this improves patient access, is inferred to improve patient outcomes through earlier intervention in their health condition, releases medical specialist time (to perform surgeries or see complex patients), and no adverse outcomes have been reported, yet the defensiveness around scope creep remains. In a health care system that is increasingly stretched while purporting to be patient-focused, these legislative barriers appear to be based on historical perspectives rather than looking forward to enabling the health care industry to use "force multipliers" effectively.

\section{Conclusion}

Despite many literature reviews indicating a lack of highquality research into the area of ESP for allied health professionals, and clear recommendations on evidence or study design required, little improvement has been noted between 2005 and 2013. Many studies have outlined tasks considered to be expanded scope, training frameworks, implementation processes, highlighted potential barriers and provided preliminary evidence in relation to positive effects in terms of ability to perform extended scope tasks, patient outcomes, patient satisfaction, and cost benefits. If one were to take a Bayesian perspective when reviewing the evidence thus far, it appears that ESP allied health practitioners could be a cost-effective, consumer-accepted investment that health services can make to improve patient outcomes.

\section{Disclosure}

The authors report no conflicts of interest in this research.

\section{References}

1. ONU. World population ageing 1950-2050. 2002. Available from: www. un.org/esa/population/publications/worldageing19502050/.

2. Pieper C, Kolankowska I. Health care transition in Germany standardization of procedures and improvement actions. J Multidiscip Healthc. 2011;4:215-221.
3. Lubitz J, Cai L, Kramarow E, Lentzner H. Health, life expectancy, and health care spending among the elderly. $N$ Engl J Med. 2003;349: $1048-1055$.

4. Schoen C, Osborn R, How SK, Doty MM, Peugh J. In chronic condition: experiences of patients with complex health care needs, in eight countries, 2008. Health Aff (Millwood). 2009;28:w1-w16.

5. Goss JR. Projection of Australian health care expenditure by disease, 2003 to 2033. Canberra, Australia: Australian Institute of Health and Welfare; 2008. Available from: http://www.aihw.gov.au/publications/ hwe/pahced03-33/pahced03-33.pd. Accessed August 18, 2014.

6. Australian Institute of Health and Welfare. Public health expenditure in Australia 2008-2009. Canberra, Australia: Australian Institute of Health and Welfare; 2011. Available from; http://www.aihw.gov.au/publicationdetail/?id=10737418329\&tab=1. Accessed August 18, 2014.

7. Gill PS. Technological innovation and its effect on public health in the United States. J Multidiscip Healthc. 2013;6:31-40.

8. Gray LC, Wright OR, Cutler AJ, Scuffham PA, Wootton R. Geriatric ward rounds by video conference: a solution for rural hospitals. Med J Aust. 2009;191:605-608.

9. Chopra M, Munro S, Lavis JN, Vist G, Bennett S. Effects of policy options for human resources for health: an analysis of systematic reviews. Lancet. 2008;371:668-674.

10. Duckett SJ. Health workforce design for the 21 st century. Aust Health Rev. 2005;29:201-210.

11. Gilmore LG, Morris J, Murphy K, Grimmer-Somers K, Kumar S. Skills escalator in allied health: a time for reflection and refocus. J Multidiscip Healthc. 2011;3:53-58.

12. Holdsworth LK, Webster VS, McFadyen AK. Physiotherapists' and general practitioners' views of self-referral and physiotherapy scope of practice: results from a national trial. Physiotherapy. 2008;94:236-243.

13. Kersten P, McPherson K, Lattimer V, George S, Breton A, Ellis B. Physiotherapy extended scope of practice - who is doing what and why? Physiotherapy. 2007;93:235-242.

14. Kersten SG, Lattimer V, Ellis B. Briefing paper: Extending the practice of allied health professionals in the NHS. Available from: http://www.nets. nihr.ac.uk/_data/assets/pdf_file/0005/81248/BP-08-1203-031.pdf.

15. McPherson K, Kersten P, George S, et al. A systematic review of evidence about extended roles for allied health professionals. $J$ Health Serv Res Policy. 2006;11:240-247.

16. McPherson K, Kersten P, George S, et al. Extended roles for allied health professionals in the NHS. 2004. Available from: http://www.nets.nihr. ac.uk/_data/assets/pdf_file/0019/64342/FR-08-1203-031.pdf.

17. Sibbald B, Shen J, McBride A. Changing the skill-mix of the health care workforce. J Health Serv Res Policy. 2004;9 Suppl 1:28-38.

18. Stanhope J, Grimmer-Somers K, Milanese S, Kumar S, Morris J. Extended scope physiotherapy roles for orthopedic outpatients: an update systematic review of the literature. J Multidiscip Healthc. 2012;5:37-45.

19. Lundon K, Shupak R, Sunstrum-Mann L, Galet D, Schneider R. Leading change in the transformation of arthritis care: development of an interprofessional academic-clinical education training model. Healthc $Q$. 2008; 11:62-68.

20. Aiken AB, Atkinson M, Harrison MM, Hope J. Reducing hip and knee replacement wait times: an expanded role for physiotherapists in orthopedic surgical clinics. Healthc Q. 2007;10:88-91.

21. Aiken AB, Harrison MM, Hope J. Role of the advanced practice physiotherapist in decreasing surgical wait times. Healthc Q. 2009;12: $80-83$.

22. Boissonnault WG, Badke MB, Powers JM. Pursuit and implementation of hospital-based outpatient direct access to physical therapy services: an administrative case report. Phys Ther. 2010;90:100-109.

23. Crane J, Delany C. Physiotherapists in emergency departments: responsibilities, accountability and education. Physiotherapy. 2013;99: 95-100.

24. McPherson KM, Reid DA. New roles in health care: what are the key questions? Med J Aust. 2007;186:614-615. 
25. Robarts S, Kennedy D, MacLeod AM, Findlay H, Gollish J. A framework for the development and implementation of an advanced practice role for physiotherapists that improves access and quality of care for patients. Healthc Q. 2008;11:67-75.

26. Merlin T, Weston A, Tooher R. Extending an evidence hierarchy to include topics other than treatment: revising the Australian 'levels of evidence'. BMC Med Res Methodol. 2009;9(1):34.

27. Aiken AB, Harrison MM, Atkinson M, Hope J. Easing the burden for joint replacement wait times: the role of the expanded practice physiotherapist. Healthc Q. 2008;11:62-66.

28. MacKay C, Davis A, Mohamed N, Badley E. Physical therapists working in expanded roles in orthopaedic clinics: impact on non-surgical patients with arthritis. Osteoarthritis Cartilage. 2012;20:S166.

29. Lineker SC, Lundon K, Shupak R, Schneider R, Mackay C, Varatharasan N. Arthritis extended-role practitioners: impact on community practice (an exploratory study). Physiother Can. 2011;63: 434-442.

30. Grimmer-Somers K, Morris J, Murphy K. Multidisciplinary musculoskeletal triage clinic: outcomes 12 months on. Scientific poster RHPA-P3 presented at the Australian Rheumatology Association annual meeting, May 12-15, 2012, Canberra, Australia.

31. Kennedy DM, Robarts S, Woodhouse L. Patients are satisfied with advanced practice physiotherapists in a role traditionally performed by orthopaedic surgeons. Physiotherapy Canada. 2010;62(4):298-305.

32. Passalent LA, Kennedy C, Warmington K, et al. System integration and clinical utilization of the Advanced Clinician Practitioner in Arthritis Care (ACPAC) program-trained extended role practitioners in Ontario: a two-year, system-level evaluation. Healthc Policy. 2013;8: 56-70.

33. Rhon DI. A physical therapist experience, observation, and practice with an infantry brigade combat team in support of Operation Iraqi Freedom. Mil Med. 2010;175:442-447.

34. Kilner E. What evidence is there that a physiotherapy service in the emergency department improves health outcomes? A systematic review. J Health Serv Res Policy. 2011;16:51-58.

35. Li LC, Westby MD, Sutton E, Thompson M, Sayre EC, Casimiro L. Canadian physiotherapists' views on certification, specialisation, extended role practice, and entry-level training in rheumatology. $B M C$ Health Serv Res. 2009;9:88.

36. Green A, Perry J, Harrison K. The influence of a postgraduate clinical master's qualification in manual therapy on the careers of physiotherapists in the United Kingdom. Man Ther. 2008;13:139-147.

37. Braund R, Abbott JH. Recommending NSAIDs and paracetamol: a survey of New Zealand physiotherapists' knowledge and behaviours. Physiother Res Int. 2011;16:43-49.

38. McClellan C, Greenwood R, Benger J. Effect of an extended scope physiotherapy service on patient satisfaction and the outcome of soft tissue injuries in an adult emergency department. Emergency Medicine Journal. 2006;23(5):384-387.
39. Desmeules F, Roy J-S, MacDermid JC, Champagne F, Hinse O, Woodhouse LJ. Advanced practice physiotherapy in patients with musculoskeletal disorders: a systematic review. BMC Musculoskelet Disord. 2012;13:107.

40. Stanhope J, Beaton K, Grimmer-Somers K, Morris J. The role of extended scope physiotherapists in managing patients with inflammatory arthropathies: a systematic review. Open Access Rheumatology: Research and Reviews. 2012. Available from: http://www.dovepress. com/the-role-of-extended-scope-physiotherapists-in-managing-patientswith--peer-reviewed-article-OARRR. Accessed August 19, 2014.

41. Anaf S, Sheppard LA. Physiotherapy as a clinical service in emergency departments: a narrative review. Physiotherapy. 2007;93:243-252.

42. Kilner E, Sheppard L. The 'lone ranger': a descriptive study of physiotherapy practice in Australian emergency departments. Physiotherapy. 2010;96:248-256.

43. Lundon K, Shupak R, Reeves S, Schneider R, McIlroy JH. The Advanced Clinician Practitioner in Arthritis Care program: an interprofessional model for transfer of knowledge for advanced practice practitioners. J Interprof Care. 2009;23:198-200.

44. Amaker RJ, Brower KA, Admire DW. The role of the upper extremity neuromusculoskeletal evaluator at peace and at war. $J$ Hand Ther. 2008;21:124-128.

45. Williamson GR, Webb C, Abelson-Mitchell N, Cooper S. Change on the horizon: issues and concerns of neophyte advanced health care practitioners. J Clin Nurs. 2006;15:1091-1098.

46. Mathisen BA, Carey LB, O’Brien A. Incorporating speech-language pathology within Australian neonatal intensive care units. J Paediatr Child Health. 2012;48:823-827.

47. Anaf S, Sheppard LA. Lost in translation? How patients perceive the extended scope of physiotherapy in the emergency department. Physiotherapy. 2010;96:160-168.

48. Davis S, Machen MS, Chang L. The beneficial relationship of the colocation of orthopedics and physical therapy in a deployed setting: Operation Iraqi Freedom. Mil Med. 2006;171:220-223.

49. Humphreys A, Richardson J, Stenhouse E, Watkins M. Assessing the impact of nurse and allied health professional consultants: developing an activity diary. J Clin Nurs. 2010;19:2565-2573.

50. MacKay C, Davis AM, Mahomed N, Badley EM. Expanding roles in orthopaedic care: a comparison of physiotherapist and orthopaedic surgeon recommendations for triage. J Eval Clin Pract. 2009;15: 178-183.

51. Pearse E, Maclean A, Ricketts D. The extended scope physiotherapist in orthopaedic out-patients - an audit. Ann R Coll Surg Engl. 2006;88:653.

52. Rhon DI, Gill N, Teyhen D, Scherer M, Goffar S. Clinician perception of the impact of deployed physical therapists as physician extenders in a combat environment. Mil Med. 2010;175:305-312.

53. Bethel J. The role of the physiotherapist practitioner in emergency departments: a critical appraisal. Emerg Nurse. 2005;13:26-31. 


\section{Supplementary material}

\section{Search terms list}

advanc* practi* OR consultant therapist* OR cross boundar* OR current role* OR enhan* practice* OR enhan* scope* OR existing role* OR existing scope* OR exp* practice* OR expan* scope* ORext* scope* OR exten* practice* OR interdisciplinary collaboration OR joint practice* OR multi* task* OR new role* OR new scope* OR physician exten* OR physician assist* OR profession* boundar* OR role* boundar* OR role* chang* OR role* collaborati* OR role* cross* OR role* defin* OR role* demarcation* OR role* ehan* OR role* expan* OR role* exten* OR role* interdisciplin* OR role* interprofessional* OR role* modern* OR role* overlap* OR role* professional* OR role* redefin* OR role* shar* OR role* shift* OR scope of practice OR shar* competenc* OR shift* boundar* OR skill interdisciplin* OR skill* overlap* OR skill* shar* OR specialist practitioner* OR traditional role* OR transdisciplinary practice* OR consultant practitioner* OR clinical specialist* OR int??disciplinary competenc* OR int??disciplinary practice*

Exercise therap* OR kinesiotherap* OR manual therap* OR physiotherapy OR physical therap* OR physio OR physios OR physiotherapy* OR ergotherap* OR occupational therap* OR recreational therap* audiolog* OR auditory rehabilitation OR language therap* OR logopaed* OR rehabilitation of speech and language disorders OR speech and language OR speech and language pathologist* OR speech and language rehabilitation OR speech language pathologist* OR speech language therap* OR speech pathology* OR speech rehabilitation OR speech therap* OR speech train*

Nuclear medicine OR radiographer* OR radiography OR radiologic* technician* OR radiologic* technologist* OR radiology personnel OR technology radiologic OR technology-radiologic OR air ambulance* OR ambulance paramedic* OR ambulance personnel OR ambulance staff* OR ambulance technician* OR crisis intervention OR critical care OR emergenc* medic* OR emergency care OR emergency health OR emergency personnel OR emergency service* OR emergency treatment* OR first aid* OR paramedic* OR rapid response* OR resuscitation OR urgent transport* OR d?spatch* OR priority d?spatch

Allied health OR generic therapist* OR health manpower OR health occupation* OR health personnel OR multiprofessional* OR profession* allied to health OR profession* allied to medicine OR rehabilitati*

\section{Publish your work in this journal}

The Journal of Multidisciplinary Healthcare is an international, peerreviewed open-access journal that aims to represent and publish research in healthcare areas delivered by practitioners of different disciplines. This includes studies and reviews conducted by multidisciplinary teams as well as research which evaluates the results or conduct of such teams or healthcare processes in general. The journal covers a wide range of areas and welcomes submissions from practitioners at all levels, from all over the world. The manuscript management system is completely online and includes a very quick and fair peer-review system. Visit http://www.dovepress.com/testimonials.php to read real quotes from published authors. 